\title{
Comunicación

\section{Diversidad audiovisual e integración cultural: analizando el programa Ibermedia}

JOSÉ MANUEL MORENO DOMÍNGUEZ1

Pese a deficiencias en el funcionamiento y a su falta de ambición económica, la creación del programa Ibermedia ha sido un punto de inflexión para crear un espacio audiovisual común para la región iberoamericana. A través de un fondo financiero multilateral, el programa ha intentado estimular la coproducción de productos para el cine y la televisión, el montaje inicial de proyectos cinematográficos, la distribución de películas dentro del mercado regional y la formación de recursos humanos para la industria audiovisual. Realizar una primera evaluación de sus primeros años de funcionamiento (1998-2007), analizar las opiniones que se han vertido sobre el mismo, así como realizar una primera valoración de su impacto en la región es el objetivo central de este trabajo.

PALABRAS CLAVE: industria audiovisual, políticas culturales, diversidad cultural, Ibermedia.

1 Universidad de Sevilla, España.

Correo electrónico: jmmoreno@comunicacionydesarrollo.org
The 'Ibermedia' program, launched in 1997 on the V Cumbre Iberoamericana de Jefes de Estado y de Gobierno, has laid the foundations for future and effective audiovisual collaboration in the region, an effort that could have been much more fruitful had it been designed with better resources and more ambitious aims in mind. Today, a total number of thirteen countries interact through 'Ibermedia', using a multilateral fund scheme in order to promote not only co-productions but also initiatives in sectors such as film distribution, advertising campaigns for concrete films or professional training. This paper will undertake an assessment of the program's first functioning years (1998-2007), analyzing comments and opinions on 'Ibermedia', as well as making an evaluation of its impact in the region.

KEY WORDS: audiovisual industry, cultural policies, cultural diversity, Ibermedia. 
Para salvarnos, juntarnos. Como los dedos en la mano. Como los patos en el vuelo.

Eduardo Galeano

\section{INTRODUCCIÓN}

En los últimos años nuestras sociedades están sufriendo un sensible cambio propiciado por las transformaciones sociales que han generado el desarrollo de las nuevas tecnologías, la internacionalización de los mercados y las transacciones financieras. Esta nueva "sociedad global" ha levantado un amplio debate sobre los aspectos culturales que se mueven en dos lógicas aparentemente contradictorias: la de una homogenización, cada vez mayor, como principal elemento favorable de cara a los intereses de las empresas transnacionales que comercian con ellos y, al mismo tiempo, la diversificación de identidades a través de un proceso de recuperación territorial y reconstrucción de rasgos y valores comunes tradicionales.

No obstante, y como defienden diferentes autores (García Canclini, 2002; Ortiz, 1994; Martín-Barbero, 2002), el ámbito de la cultura contiene una complejidad especial dentro del discurso en boga de la globalización que no podemos limitar a la dialéctica entre un planeta interconectado por bienes culturales y mensajes homogéneos procedentes de Norteamérica, frente a la diversidad de situaciones y culturas locales de cada región. Lo cierto es que muchas veces lo que denominamos como globalización se concreta en un agrupamiento regional por razones de afinidad geográfica e histórica o de acceso diferencial a los recursos económicos y tecnológicos, y que el imaginario mundializado que nos ha deparado el siglo XX (a través de las nuevas tecnologías, el cine o la televisión) no sólo se impone sino que convive con otras visiones del mundo entre las que se establecen jerarquías, conflictos, acomodaciones, readaptaciones, etc.

En este sentido, vivimos un momento donde esta situación y las propias dinámicas de mercado intensifican la importancia de espacios de integración y redes de cooperación transnacionales que, sustentadas en elementos sociales y culturales comunes, puedan obtener ventajas 
comparativas en terrenos claves del nuevo mercado global que garanticen un mayor equilibrio en el aprovechamiento y flujo de los recursos culturales y comunicativos, así como una mayor diversidad de formatos y contenidos.

Son en este contexto las industrias culturales las que juegan un papel fundamental por un doble motivo: en primer lugar, por la capacidad de definición de nuevos imaginarios comunes y de creación de lazos y sentidos de pertenencia ciudadana, y en segundo, por el nuevo papel de sector económico emergente que está acaparando movimientos de capital, personas y bienes culturales de gran valor para el mercado. Por eso no es de extrañar que estas industrias, en especial la de las comunicaciones, se hayan convertido en

una de las zonas de mayor competitividad y conflictividad entre intereses públicos y privados, entre países desarrollados y periféricos, y aun entre modalidades diversas de desarrollo cultural (García Canclini, 2002).

\section{POLÍTICAS PARA LA INTEGRACIÓN, POLÍTICAS PARA EL AUDIOVISUAL}

De este modo, la construcción de Iberoamérica pasa sin lugar a dudas por potenciar las diferentes industrias culturales de una región que, pese a su potencialidad como espacio de diversidad y riqueza cultural, así como por tamaño de su territorio y número de habitantes, se encuentra en muchos casos minorizada por la ocupación de grandes empresas transnacionales que controlan circuitos turísticos, musicales, editoriales o audiovisuales. Es por ello que el espacio iberoamericano de la cultura, aún por desarrollar dentro de la Sociedad de la Información, empieza a ser visto por los gobiernos como un reto y una oportunidad inigualables de desarrollo económico y social que parta del imaginario históricocolectivo que engloba a lo iberoamericano, que se enriquece desde la diversidad cultural y el mestizaje que lo compone, y que genere un mercado de intercambio compartido aproximadamente por quinientos millones de personas de habla portuguesa y castellana.

El campo del audiovisual es quizás el más evidente y, al mismo tiempo, urgente de afrontar ya que, a pesar de ser un sector estratégico no 
sólo desde el punto de vista la cultura sino también para la economía ${ }^{2}$ y la convergencia tecnológica, se encuentra completamente dependiente de productos externos (procedentes en su mayoría de la industria de los Estados Unidos) y controlado por grandes grupos multimedia. Si nos centramos en la industria cinematográfica y según apunta un informe de la UNESCO $^{3}$ hecho público en 2001, la mayor parte de los países del subcontinente latinoamericano se encuentran catalogados como zonas de producción baja, con menos de 20 películas al año. Estos países basan su oferta de forma casi exclusiva en la importación de productos extranjeros (principalmente procedentes de Hollywood), destacando el caso extremo de Nicaragua, que no consigue producir ni tan siquiera un filme al año. Tan sólo Brasil y Argentina ${ }^{4}$ logran superar la barrera del subdesarrollo audiovisual, apoyándose en medidas de protección para los productos nacionales y en la amplitud de sus mercados internos, pero en general las cotas de dominación norteamericana llegan a una media de $80 \%$ del total de las películas exhibidas, alcanzando hasta 95\% en países como Costa Rica o Ecuador.

En este sentido, los países de América Latina no han podido desarrollar los mismos mecanismos de defensa que otras cinematografías como la europea frente a estas amenazantes cifras, fundamentalmente por la imposibilidad de implicar al Estado en la financiación de la industria audiovisual que, en la mayoría de los casos, ha sido considerada, como bien señala John Hill (1998), un "lujo del que se podía prescindir".

Además, la escasa integración regional del mercado audiovisual latinoamericano y, sobre todo, la falta de recursos para exportar, han impedido que las películas de distintos países traspasaran las fronteras nacionales y las del propio continente, lo que lleva a Enrique Sánchez Ruiz (2001) a afirmar que los países latinoamericanos arrastran una situación

2 En el año 2000 se estimaba en más de 20000 millones de dólares los recursos que generaba esta industria en Iberoamérica.

3 UNESCO “A survey on national cinematography” disponible en: http://www. unesco.org/culture/industries/cinema/html_eng/survey.shtml

4 Ambos países conseguían en 2004 tener una presencia de espectadores en el cine nacional cercana a $15 \%$ del total, y colocaban una película propia entre las diez más vistas del país. 
histórica donde no están acostumbrados a exportar, intercambiar y exhibir sus productos cinematográficos, como prueba un estudio de Media Research \& Consultancy-Spain, donde se recogía que tan sólo $2 \%$ del dinero recaudado en taquilla dentro del ámbito iberoamericano, pertenecía a películas originarias de la región y los datos del Observatorio Europea del Audiovisual, que otorga a las películas latinoamericanas un casi insignificante $0,14 \% 5$ del mercado europeo (correspondiendo más de la mitad de esa cuota a la distribución específica en España).

Esta situación ha estado reforzada, igualmente, por la propia dinámica de la televisión en Latinoamérica que abrazó históricamente un modelo comercial que debilitó el sentido de los servicios públicos y que constituyó una amalgama de situaciones nacionales diversas que no llegaron a establecer relaciones intrarregionales, motivos por los cuales no se consiguió construir las bases de una industria sólida de programas audiovisuales que apoyasen las producciones cinematográficas, al mismo tiempo que facilitasen su distribución y exhibición.

Ante este panorama, parecía evidente y prioritaria la necesidad de abrir un espacio audiovisual iberoamericano integrado, que pudiera favorecer las industrias locales frente a las grandes multinacionales, incentivar las coproducciones y dar facilidades para la distribución y la exhibición de los productos autóctonos.

Sin embargo, como señala Rafael Roncagliolo (1996), los esfuerzos por conseguir una integración iberoamericana en el campo de las comunicaciones han sido hasta el momento "una lista de intentos fallidos", ya sean en el sector de las agencias de prensa, de los organismos interinstitucionales o de las asociaciones de medios y/o profesionales. En lo que se refiere al ámbito audiovisual existe un precedente muy interesante que llevó a cabo la empresa pública brasileña "Embrafilme", cuando en 1977 propuso la creación de "un mercado común latinoamericano del cine" que incluyese a todos los países hispanos y lusoparlantes. Angola, Argentina, Brasil, Colombia, México, Perú, Uruguay, Venezuela y tam-

5 Datos extraídos de la nota de prensa que emitió el Observatorio Audiovisual Europeo sobre la distribución de filmes procedentes de terceros países en la Unión Europea (1996-2002). Disponible en http://www.obs.coe.int/about/ oea/pr/disfilms_pays_tiers.html 
bién España y Portugal, incluso llegaron a mantener reuniones formales para discutir la cuestión, aunque finalmente tanto la disparidad entre las cinematografías de estos países como la falta de objetivos comunes dieron al traste con este proyecto.

No obstante, si dejamos atrás experiencias ya muy antiguas en el tiempo y en las circunstancias sociales como la de "Embrafilme" o la de la Unión de la Cinematografía Hispanoamericana (UCHA), las relaciones en materia audiovisual de los países iberoamericanos se han limitado en las últimas décadas a restringidos acuerdos particulares (poco desarrollados y en muchos casos incumplidos) entre varios países que se comprometían a apoyar como propias las coproducciones que se realizasen en este marco. Este era el caso de los acuerdos bilaterales de España con Portugal y con México, de Brasil con Portugal y Argentina, o de Chile y Venezuela por citar algunos de los más importantes.

Sin embargo, en el umbral de la década de los noventa se van a dar dos circunstancias que, promovidas por instancias políticas, van a convertirse en cruciales de cara a retomar el debate y el interés por acercar el espacio audiovisual de la Península Ibérica con el de los países de América Latina:

1) La puesta en marcha, el 11 de noviembre de 1989 en Caracas, del primer Convenio de Integración Cinematográfica Iberoamericana que diseñará un proyecto de integración entre los países de la región para, mediante una participación equitativa, reactivar y armonizar sus políticas cinematográficas. Los miembros integrantes de este convenio fueron Argentina, Bolivia, Brasil, Colombia, Cuba, Ecuador, México, Nicaragua, Panamá, Perú, España y Venezuela. El Convenio daba lugar a la creación de dos órganos que en la década de 1990 se convirtieron en el epicentro de la actividad audiovisual iberoamericana, fomentando el sector a través de la consolidación de este espacio comercial, a la vez que creando una identidad desde los valores simbólicos y de autorreconocimiento de la cinematografía. Estos dos órganos fueron: la Conferencia de Actividades Cinematográficas de Iberoamérica (CACI) y la Secretaría Ejecutiva de la Cinematografía Iberoamericana (SECI), actuando el primero de ellos como órgano máximo y el segundo con funciones de apoyo técnico y ejecutivo. 
Desde entonces la CACI, pese a periodos de actividad irregular, ha ampliado sus miembros a trece y sigue siendo la principal institución que vela por el desarrollo audiovisual de la región y pretende consolidar la integración cinematográfica a través de mecanismos de intercambio y cooperación multilateral, basados en el núcleo de la identidad cultural como método de fortalecimiento común.

2) La institucionalización de las Conferencias Iberoamericanas de Jefes de Estado y de Gobierno que se iniciaron en Guadalajara (México) en 1991, y que desde su segunda edición empezó a concertar programas de cooperación de diversa índole (alfabetización, intercambios educativos, fondos de ayuda para la población indígena, etc.). En esta segunda cumbre celebrada en Madrid sería donde quedaría aprobado, como primera medida importante dentro del sector audiovisual, el Programa sobre Televisión Educativa Iberoamericana.

Estos encuentros fijaron la cultura, la educación y la innovación tecnológica como los grandes retos sobre los que se debería concentrar los esfuerzos a partir de proyectos concretos de cooperación. Sería, de esta forma, en la V Conferencia celebrada en San Carlos de Bariloche (Argentina) donde se presenta por primera vez el borrador de un proyecto futuro para fomentar un espacio audiovisual iberoamericano al que se denominó Ibermedia.

Por otra parte, fuera de estas acciones de ámbito político también se producía la necesaria convergencia de productores y profesionales del sector que comenzaban a sentir la necesidad de entablar relaciones de ámbito internacional. En este sentido, experiencias como la del Festival Internacional del Nuevo Cine Latinoamericano que se celebra en la Habana o el Festival Iberoamericano de Huelva, se convirtieron en pioneras y, al mismo tiempo, en reuniones informales de todo el sector de la cinematografía iberoamericana.

Podemos destacar asimismo una experiencia española que, a través de la Asociación Nacional de Empresas (ANEPA) y la Federación de Asociaciones de Productores Audiovisuales Españoles (FAPAE) se viene desarrollando desde 1996: el llamado MIDIA o Mercado Iberoamericano de la Industria Audiovisual. Este mercado facilita el encuentro de productores de toda Iberoamérica (con una importante participación de las televisiones) que establece un espacio de intercambio y de va- 
loración directa de futuros proyectos. Según el productor Gerardo Herrero, el mercado se ha convertido en un excelente vivero de proyectos que aspiran a beneficiarse del Fondo Ibermedia, ya que en las últimas ediciones se ha creado paralelamente un Mercado de Proyectos de Coproducción.

Por otro lado, dentro del debate de creación de Ibermedia y a partir de las reuniones de los más importantes productores de cine y televisión de los países del ámbito iberoamericano, se constituyó en 1997 la Federación Iberoamericana de Productores Cinematográficos y Audiovisuales (FIPCA) que, según su propio postulado, actúa en ámbitos nacionales e internacionales promocionando las coproducciones iberoamericanas y una correcta distribución y difusión de todos estos productos frente a la actual situación de abuso de posiciones dominantes en los mercados de cada país. Así, es importante destacar la posición que manifestaba dicha Federación en un escrito publicado en el 2002 en el marco del debate internacional sobre la "excepción cultural" del cine y la televisión, de cara a las negociaciones que se desarrollaban en la Organización Mundial del Comercio (OMC):

En los tratados comerciales, los bienes culturales no pueden ser equiparados a cualquier otro producto o servicio (...) Los bienes culturales de producción local, que cuentan nuestras historias y costumbres y enriquecen nuestro conocimiento a la vez que difunden nuestra cultura, "compiten" cada día en condiciones más desfavorables respecto a los grandes consorcios transnacionales. [Por eso] la valoración de las industrias culturales nos debe resultar tan estratégica como lo es para los Estados Unidos: así como sus gobiernos destinan recursos y esfuerzos a reclamar políticas internacionales que protejan el derecho de autor o solicitan acciones contra la piratería, los Estados Iberoamericanos necesitamos políticas que con la misma firmeza y energía fomenten nuestra producción y distribución de creaciones intelectuales. ${ }^{6}$

Lo cierto es que, si bien no comenzaría con tanta firmeza y convicción en sus pretensiones, esta dinámica de actividades y convenios comunes propició definitivamente la puesta en marcha del programa.

6 Publicado en el Suplemento Especial número 26 de INFODAC. 


\section{EL PROGRAMA IBERMEDIA (1995-2006): \\ MÁS DE UNA DÉCADA DE FUNCIONAMIENTO}

Como ya hemos señalado, Ibermedia nació como proyecto en la V Conferencia Iberoamericana de San Carlos de Bariloche, aunque se aprobó definitivamente su funcionamiento sobre la base de las decisiones adoptadas en la VII Cumbre Iberoamericana de Jefes de Estado y de Gobierno celebrada en 1997 en Isla Margarita (Venezuela). El programa, integrado dentro de la política audiovisual de la CACI y con la asesoría y participación de la FIPCA, nace como un programa de fomento a través de un fondo financiero multilateral común, que tiene como principal objetivo impulsar la industria audiovisual iberoamericana, estimulando la coproducción de productos para el cine y la televisión, además de apoyar el montaje inicial de proyectos cinematográficos, la distribución de películas en el mercado regional y la formación de recursos humanos para la industria audiovisual.

El fondo fue comprometido en un primer momento por doce países, aunque finalmente arrancaría en 1998 con la aportación de tan sólo nueve gobiernos, entre los cuales España y México aportaban más de la mitad del total (3.5 millones de dólares). Los siete restantes eran Argentina, Brasil, Colombia, Cuba, Portugal, Uruguay y Venezuela. Tras la puesta en marcha del programa se han ido sumado progresivamente otros países por este orden: Chile, Perú, Bolivia y Puerto Rico que aportan la cantidad mínima exigida (100 000 dólares).

Ibermedia está constituido por un Comité Intergubernamental (CII) y por una Unidad Técnica (UTI). El primero, en cuyo seno cada Estado miembro designa una unidad cinematográfica como su representante, se encarga de definir la política, las modalidades de obtención de la ayuda y toma todas las decisiones, mientras que la UTI asume la responsabilidad de la ejecución y el funcionamiento del programa.

El programa tiene carácter plurianual y ha cumplido ya una primera fase con una vigencia de cinco años, que fue desde enero de 1998 a diciembre de 2002, tiempo este donde se nutrió económicamente con las aportaciones de cada país, así como de las devoluciones de los préstamos concedidos y de los intereses que generaron las cuentas bancarias 
abiertas. Durante este espacio temporal se concentraron las líneas de actuación en cuatro grupos diferentes:

a) Apoyo a la coproducción de películas y programas de televisión iberoamericanos.

b) Apoyo a la distribución y promoción de películas y programas de televisión.

c) Desarrollo de proyectos de cine y televisión iberoamericanos.

d) Apoyo a la formación para los profesionales de la industria iberoamericana de programas audiovisuales.

El dinero que se otorgó a cada proyecto se hizo en calidad de préstamos recuperados más tarde sobre la recaudación de la película. Únicamente las modalidades de promoción y formación recibieron un aporte a fondo perdido que en ningún caso podía exceder de un límite (30 000 US\$7 y 2500 US\$ respectivamente) ni de $50 \%$ del total previsto.

El Comité Intergubernamental, que se reúne dos veces al año, otorga cada final de año, por criterio de calidad de los proyectos y no por equidad respecto al aporte económico que cada país hace al fondo (no obstante se garantiza al menos la aprobación de un proyecto por país), una relación de ayudas que en esta primera etapa se ha distribuido como se muestra en el cuadro 1.

Si hacemos una primera interpretación de estos datos se pude apreciar claramente una tendencia al incremento del número de coproducciones apoyadas por el fondo, mientras que los proyectos elegidos para las áreas de distribución y promoción se reducen de forma sensible. En cuanto al desarrollo de proyectos y a las ayudas de formación podemos decir que han mantenido un número constante si exceptuamos el año 2000, donde se limitó el número de proyectos aprobados y casi se duplicó el número de personas financiadas para realizar algún tipo de formación audiovisual.

Para especificar aún más estos datos, podemos desglosar como ejemplo en porcentaje, la repartición económica que se hizo en 1998 y que distribuyó el fondo (3 579022 US \$), exceptuando una pequeña partida que se destinó a la estructura técnica, de esta forma: $74.2 \%$

7 En este caso, 2/3 de la ayuda se concederá en concepto de préstamo y sólo el tercio restante como subvención. 


\section{CUADRO 1}

RESULTADOS DE LAS AYUDAS OTORGADAS POR EL PROGRAMA IBERMEDIA EN SU PRIMER CICLO DE FUNCIONAMIENTO (1998-2002)

\begin{tabular}{cccccc} 
& Países & $\begin{array}{c}\text { Co- } \\
\text { producciones }\end{array}$ & $\begin{array}{c}\text { Pro- } \\
\text { yectos }\end{array}$ & $\begin{array}{c}\text { Distribución/ } \\
\text { Promoción }\end{array}$ & Formación \\
\hline 1998 & 9 & 15 & 27 & $21-21\left(^{*}\right)$ & 24 \\
1999 & 10 & 14 & 25 & $17-24$ & 27 \\
2000 & 11 & 19 & 18 & 21 & 49 \\
2001 & 12 & 26 & 23 & 11 & 30 \\
2002 & 13 & 26 & 15 & 13 & 35 \\
Total & 13 & 100 & 108 & 128 & 165
\end{tabular}

Fuente: (elaboración propia) datos extraídos de la página oficial en español del programa en la dirección: http://www.programaibermedia.com.

(*) En los dos primeros años de funcionamiento los proyectos presentados en esta área se aprobaron de forma diferenciada según estuviesen destinados a la distribución o a la promoción de películas procedentes de países iberoamericanos.

para coproducciones, 9.5 para el desarrollo de proyectos, 14.5 para distribución y promoción y 1.8 para formación audiovisual. Si tenemos en cuenta la previsión inicial que era de $60 \%, 5 \%, 30 \%$ y $5 \%$ respectivamente, comprobamos cómo desde un primer momento el programa ha centrado sus esfuerzos en el apoyo de realizaciones conjuntas.

Conseguir poner en el circuito más de cien coproducciones iberoamericanas es sin lugar a dudas un logro importante que ha movilizado nuevos contactos entre países y productores, y ha generado empleos y capitales para la industria del audiovisual. No obstante, no creemos que con las posibilidades económicas que cuenta el programa sea la base para conseguir ampliar notoriamente la circulación de las obras audiovisuales iberoamericanas en su propio mercado y en el internacional. Ya que la principal carencia que tiene la región sea quizás la de un circuito de distribución importante que apoye las producciones propias, principalmente porque el mercado de la distribución ha sido absorbido casi por completo por las llamadas majors $o$ grandes empresas norte- 
americanas que llegan en Colombia, por ejemplo, a dominar 95\% del total de películas que se distribuyen. Esto hace que las posibilidades de las pequeñas productoras sean prácticamente nulas, lo que también genera un tipo de cine absolutamente comercial que no propicia la entrada de productos de características diferentes (pequeñas producciones, cine experimental, cine de animación, etc.).

En este sentido, la estrategia en los estrenos de los filmes norteamericanos (precedidos de una amplia campaña de promoción), consiste en acaparar el mayor número de salas posibles lo que impide el acceso de otras producciones. Hollywood lo tiene claro: hay que rentabilizar los costes por lo que se debe a toda costa "conquistar al público por la fuerza de una producción, bloquear el mercado con una sola película" (citado por José Carlos Avellar y Paulo Sérgio Almeida en Toledo, 1998). Esto está, asimismo, muy ligado a la entrada de grandes empresas al mundo de la exhibición, que ha adoptado una forma completamente diferente donde las pequeñas salas o los grandes cines históricos están desapareciendo y dando lugar a complejos multiplex con gran número de pantallas. Así, mientras el total de cines disminuye en todas partes, el número de salas se hace cada vez mayor.

Estas coproducciones deberían ser en el futuro más una consecuencia del nivel de contactos e intercambios que exista entre los profesionales del medio audiovisual, así como del conocimiento mutuo entre países, que la principal vía para establecer relaciones entre productores, ya que son muy pocas las películas que acceden a estas ayudas de coproducción y, a menudo, son precisamente las que están sustentadas por productoras de mayor capacidad y que por sí mismas ya tenían establecido contactos con otras empresas internacionales.

Por otro lado, la producción propia de cada país parece que está recuperando espacio sobre las grandes producciones norteamericanas. Se ha producido una ligera revalorización del producto nacional que aglutina una cantidad creciente de espectadores en los últimos años en países como México, ${ }^{8}$ Brasil o Argentina. Este es igualmente el caso de España, que ha ampliado su porcentaje de número de espectadores para

8 A pesar de producir tan sólo 22 películas durante el 2000, las producciones mexicanas lograron $15 \%$ del total de espectadores. 
el cine español en $7 \%$ en los últimos cuatro años. Esto está permitiendo que poco a poco los productores, y en algunos casos las televisiones nacionales, apoyen estas películas y que los estrenos autóctonos estén en cierta forma revitalizando la industria del audiovisual. No obstante, esto no se siente todavía de forma considerable en la circulación de películas latinoamericanas por otros países diferentes a los de su origen, por lo que sólo ocupan entre $1 \%$ y $2 \%$ de la cuota de mercado en las salas de exhibición.

Pero para ello es muy importante la intervención de los gobiernos a través de medidas que fomenten y protejan las producciones nacionales y no dejen a estas a merced de la "mano invisible del mercado". Así, como señala el productor mexicano Jorge Sánchez, se hace imprescindible construir un espacio audiovisual propio que contemple no sólo medidas de fomento estatal a la producción, sino verdaderas "políticas que protejan esa producción en la circulación y comercialización tanto en el país de origen, como en los países de la región".

En este sentido, la afirmación del profesor Francisco Sierra sobre la situación del mercado europeo, podríamos trasladarla, incluso con características más acentuadas, a la realidad iberoamericana, donde las dificultades por contrarrestar las lógicas del mercado y las contradicciones, en la medida que se toman, se sitúan en el centro del debate político:

El circuito de distribución comercial sigue una lógica de valorización dominada por los grandes grupos multimedia transnacionales. Por ello, la confianza de la UE en el mercado al planear las políticas de apoyo a la producción audiovisual comunitaria no deja de ser una contradicción en sus términos, pues el mercado —obvio es decirlo— no es libre ni competitivo; antes bien, aparece atravesado y dominado por grupos oligopólicos que controlan no sólo los tradicionales circuitos de distribución y exhibición en salas, sino también lo nuevos espacios de los parques temáticos (Disney, Time Warner, etc.), los canales especializados por cable, los grandes operadores de telecomunicación y, por supuesto, los medios audiovisuales convencionales (Sierra Caballero, 2002: 38).

En esta línea, podemos decir que la labor de las televisiones -sea cual sea su forma de transmisión (satélite, cable, etc.)- se antoja muy 
importante en lo que se refiere al apoyo del audiovisual iberoamericano, por cuanto ya sea como productores o como exhibidores facilitan la producción cinematográfica como forma de inversión muy rentable para ellos en el mundo actual. Por otro lado la televisión, como ya hemos analizado, marca en muchos casos la pauta de los gustos del público y fija un imaginario colectivo que se inserta dentro de cada sociedad. En este sentido, el programa Ibermedia parece haber centrado su atención en la producción cinematográfica y dejado de lado el mundo, si cabe, más complejo de la televisión. Si bien se recoge en las bases del programa la posibilidad de apoyar tanto coproducciones como proyectos televisivos, en la realidad estos han sido casi excepcionales y sólo encontramos uno que otro documental avalado por el fondo.

Este hecho merece sin duda una mínima reflexión que nos lleva a pensar en algunas características diferenciales de la televisión y el cine, tanto en lo que se refiere a la industria como al lenguaje de cada uno. La industria cinematográfica, de la mano de Estados Unidos, ha sabido demostrar la capacidad de universalización de sus productos, así como la mayor ramificación de su industria, que ha sido capaz de integrar sus producciones en las industrias del video doméstico, la música o el ocio. Por su parte la televisión ha generado productos más locales y menos duraderos (cosa evidente en los programas en directo), además de trasladar una imagen más banal. Quizás algunas de estas razones influyen a la hora de entender por qué regiones como la Unión Europea o Iberoamérica, en este caso, son capaces de poner en marcha proyectos de ayuda en el ámbito de la cinematografía con el aporte económico de diferentes países, mientras que las dificultades para articular redes de cooperación televisiva son, hasta el momento, casi insalvables.

No obstante, en la última Conferencia Iberoamericana de Cultura que se celebró en Córdoba (España) en junio de 2005, se abordó ampliamente esta cuestión y se aprobaron las propuestas de crear una "Televisión Cultural Iberoamericana" y un fondo multilateral que pueda estimular la coproducción y la realización de contenidos televisivos de alta calidad, así como la formación de profesionales que trabajarían en este proyecto. La propia declaración final insistía en que "dicho fondo debería considerar el exitoso programa Cumbre Ibermedia, y coordinarse con él en lo posible" 


\section{VALORACIONES Y PERSPECTIVAS DE FUTURO}

La acogida del programa Ibermedia ha sido bastante positiva en líneas generales en todo el universo iberoamericano y ha sido uno de los programas abanderados de la integración cultural que proponen teóricamente los jefes de Estado y de Gobierno de la región. En este sentido, el secretario de Estado para la Cooperación Internacional y para Iberoamérica, Miguel Ángel Cortés, hacía balance, tras estos primeros cinco años de funcionamiento, de un llamamiento a las autoridades competentes para que respondiesen a la eficacia del programa "con un mayor respaldo presupuestario y una mejor coordinación política de cara a que este espacio cultural, en español y portugués, sea cada vez más intenso". ${ }^{9}$

Sin embargo, en las propias palabras del secretario de Estado se encuentran dos de los principales cuestionamientos que ha tenido el programa durante estos primeros años:

1) La escasez de recursos del programa, que pone a disposición de las candidaturas seleccionadas cifras poco considerables en una industria tan cara como la cinematográfica.

2) Las dificultades de coordinación política y gestión provocadas por el estatuto inseguro e incierto de la CACI y por la disfuncionalidad de coordinación a través de dos sedes tan distantes como Madrid y Caracas.

Para resolver ambas cuestiones, las autoridades políticas han prometido una ampliación de los recursos económicos y una mayor estabilidad jurídica y operacional, insertando el programa dentro de la estructura de funcionamiento de la SECIB, como retos inaplazables de cara a mejorar cualitativa y cuantitativamente el programa en el futuro.

No obstante, el éxito del programa se puede avalar si consideramos la rapidez y eficiencia con la que se puso en marcha, con los más de 750 proyectos que han recibido ayuda y con el relativo éxito de circulación de algunas de las coproducciones apoyadas. En este sentido, películas como Plata quemada o Kamchatka (Argentina), En la puta vida (Uruguay) Machuca (Chile) o El crimen del Padre Amaro (Méxi-

9 Rueda de prensa celebrada en Madrid el 31 de septiembre de 2003 para presentar los resultados de esta primera etapa. 
co) han sido títulos que han recibido excelentes críticas y que se han podido ver en los mejores festivales del mundo (incluyendo la enorme repercusión de las nominaciones a los Oscar de Hollywood que tuvo la cinta mexicana).

Prueba del éxito es la continuidad en el crecimiento de países que se quieren sumar al mismo, principalmente países pequeños que, gracias a las ayudas de Ibermedia pueden incentivar una industria cara pero emergente y estratégica como la del audiovisual. Según su directora Elena Vilardell, el programa tiene actualmente sobre la mesa las solicitudes de ingreso de Costa Rica, Ecuador o Paraguay, y acaba de sumar para la convocatoria de 2006 a Panamá.

Asimismo, no es de extrañar que países europeos, como Francia e Italia, también hayan manifestado su interés por el programa ya que, según datos del Observatorio Europeo del Audiovisual, el interés de este continente por las producciones hispanoamericanas ha aumentado considerablemente. Prueba de ello son las 91 películas de la región que se estrenaron entre 1996 y 2002 y que alcanzaron una audiencia de casi 10 millones de espectadores (5 millones en España, 1350000 en Francia y 650000 en Italia). Por este motivo, la dirección de Ibermedia ya ha iniciado contactos con la Comisión Europea para establecer acuerdos parciales entre ambas regiones (tal vez ligados a los programas europeos Media y Eurimages).

Para el productor mexicano Jorge Sánchez, "Ibermedia ha sido lo más relevante que ha ocurrido en los últimos tiempos en relación con el cine hablado en español y portugués", y afirma desde su experiencia que:

"Lo esencial del programa es que requiere que los productores se vean las caras y establezcan relaciones que derivarán en un proyecto específico que incidirá en sus correspondientes mercados. Por ejemplo un país tan fuerte como Brasil no participaba en coproducciones con América Latina y ahora en Ibermedia presentaron nueve proyectos, eso indica cómo ha ascendido ese volumen. Para nosotros es importante, porque te obliga a hacerte crecer, a entrar en relaciones, que el proyecto esté muy bien presentado, aprender a escuchar y negociar". 10

10 Entrevista publicada en el diario La Jornada el 10 de octubre de 1999. 
No obstante, para el cineasta brasileño Jom Tob Azulay11 la colaboración tanto de Portugal como, especialmente, de Brasil es todavía exigua, lo que provoca la centralidad hispana del programa, por lo que considera que su país, en el marco de una nueva revitalización de MERCOSUR, debería realizar una mayor aportación económica que acabase con la tradición histórica de indiferencia de este país con América Latina, y que protegiese y fomentase la lusofonía dentro de la región. En este sentido, si sumamos las producciones de las que han formado parte empresas brasileñas o portuguesas no llegan a $25 \%$, además, en muchos casos, estas han participado como accionistas minoritarios, por lo que el número de películas de habla portuguesa es aún menor, tan sólo quince. Una cifra muy pequeña si atendemos al total de las 100 coproducciones que recibieron ayuda durante este primer periodo. En este sentido, Portugal abrió, a partir de la Constitución de la Comunidade de Países de Língua Oficial Portuguesa (julio de 1996), una iniciativa importante donde los medios eran reconocidos como instrumentos de vital importancia para su desarrollo y reconocimiento mutuo. Algunas de las medidas que se tomaron fueron la puesta en marcha de la RDP y RTP África, el proyecto de poner en marcha una RTP Brasil o el programa luso-brasileiro de coproducciones.

En un ámbito más general de valoración del programa, tampoco han faltado voces muy críticas que se han quejado por la escasez de las ayudas económicas o por la complicación en el trámite burocrático de los proyectos, que provocan que sólo las grandes productoras puedan elaborarlos con rigor y detalle. Al igual que desde algunos lugares se ha generado un cierto escepticismo sobre el programa, ya que España fue desde un primer momento la gran impulsora del mismo, y se vislumbra en este interés una visión comercial por parte del gobierno español, que pretendería con este programa, únicamente, ampliar el mercado de su cinematografía.

Este es el caso del chileno Rodrigo Díaz, asesor de cine iberoamericano de diferentes festivales europeos, que opina que los programas de cooperación no son más que una forma de inversión, una manera de poder llegar a determinados países y con esto establecer relaciones favorables para el desarrollo del país que promueve la cooperación. Según Díaz

11 Artículo publicado en el diario brasileño Estado el 17 de octubre de 2002. 
El convenio sería perfecto si se aumentara en doce o quince millones de dólares (...) pero con las ayudas que se destinan es ridículo...es un programa que está vendiendo a España en un sector privilegiado como la cultura (...) Es decir, yo para pagar una hoja en un diario importante de Europa, me cuesta 100 mil dólares y ellos venden esto en un continente completo $\mathrm{y}$, peor aún, queda una sensación muy positiva y de gratitud. ${ }^{12}$

No obstante la opinión generalizada, pese a las dudas que siempre genera una iniciativa nueva inserta en un ámbito de frustraciones históricas, ha sido la de acoger con interés y motivación las posibilidades nuevas que ha traído el programa para el campo del audiovisual en Iberoamérica y, como hemos visto, ha servido de catalizador de otras iniciativas surgidas en la región. No por ello debemos dejar de actualizar y analizar críticamente el programa para que cumpla con las expectativas levantadas, así como para que atienda a las nuevas necesidades que se plantean en este campo.

En este sentido, la puesta en marcha de un nuevo ciclo que comenzó en 2003, parece evidenciar que el programa pretende crecer cualitativa y cuantitativamente de cara a ejercer una función más eficaz dentro del mercado de la región, focalizándose en dar una mayor visibilidad a los proyectos aprobados y cumplir un nuevo objetivo que se incorpora a los principios generales del programa: el de "fomentar la integración de las empresas iberoamericanas del audiovisual en redes supranacionales". Para ello, dentro del tópico de distribución y promoción se han intentado abrir nuevas convocatorias que apoyen los proyectos dedicados a fomentar la difusión del cine iberoamericano en nuevos mercados emergentes, ya sea a través de festivales, encuentros, exposiciones, etc. Una modalidad que abre vías de colaboración con otras cinematografías para entrar en nuevos territorios, como pueden ser la Europa oriental o el continente asiático.

Igualmente, como podemos ver en el cuadro anterior, el número de producciones apoyadas se ha aumentado de forma considerable y se ha

12 Entrevista realizada en el Festival de Cartagena por Paula Marcela Trujillo. Extraída de la revista Ceropositivo en la dirección: www.ceropositivo.com/ htm/Visu/Entre/Rodrigo_Diaz.htm . 


\section{CUADRO 2}

RESULTADOS DE LAS AYUDAS OTORGADAS POR EL PROGRAMA IBERMEDIA EN SUS ÚLTIMOS TRES AÑOS (2003-2005)

\begin{tabular}{cccccc} 
& Países & $\begin{array}{c}\text { Co- } \\
\text { producciones }\end{array}$ & Proyectos & $\begin{array}{c}\text { Distribución/ } \\
\text { Promoción }\end{array}$ & Formación \\
\hline 2003 & 13 & 32 & 25 & 16 & 6 \\
2004 & 13 & 30 & 22 & 10 & 8 \\
2005 & 13 & 35 & 31 & 20 & 9 \\
Total & 13 & 97 & 78 & 46 & 23 \\
\hline
\end{tabular}

intentado administrar de mejor forma los recursos, pese a que aproximadamente $80 \%$ de los gastos del programa continúan siendo para el área de coproducciones. Así, en el área de formación se dejaron de apoyar proyectos individuales para pasar a conceder ayudas a empresas, instituciones u organizaciones de la región que fomenten la formación especializada en materia audiovisual.

No obstante, el ámbito de la distribución sigue siendo la gran asignatura pendiente y ha generado una amplia discusión en cuanto a corregir el impacto del programa en los mercados nacionales e internacionales. Algunas de las medidas que se están planteando son las siguientes: resolver si es más eficaz otorgar la ayuda a los productores para que puedan negociar de mejor manera con los distribuidores o entregarla directo al distribuidor; ampliar la convocatoria de distribución a otras áreas de explotación que puedan ser más rentables; considerar la introducción de la distribuidora como coproductora en la producción de las películas o ver la mejor manera de responder a la necesidad de que el programa fomente el desarrollo de la distribución independiente con capacidad empresarial.

Asimismo, es importante destacar el inicio del programa Cibermedia, ligado al fondo existente que priorizará los proyectos que utilicen o promuevan las nuevas tecnologías digitales del audiovisual apoyando la creación de contenidos digitales en lengua española y portuguesa, para reforzar, de este modo, la presencia iberoamericana en este sector de la economía. El problema es que esta iniciativa parte sin ningún tipo de financiación por lo que, si no se realiza un aumento presupuestario del fondo, será difícil que obtenga unos resultados importantes. 
Cibermedia se engloba dentro de un proyecto mucho más ambicioso denominado CIBERAMÉRICA, que trata de:

Crear una gran comunidad virtual iberoamericana que, utilizando como vehículo internet, refuerce los históricos lazos en las distintas áreas de actividad de la comunidad iberoamericana.

Este programa pretende subrayar e incidir directamente en lo acordado en la X Cumbre Iberoamericana de Jefes de Estado y de Gobierno, celebrada en Panamá el 17 y 18 de noviembre de 2000, en la que se concertó impulsar el desarrollo de la Sociedad de la Información y fomentar en toda Iberoamérica el uso de las nuevas tecnologías. CIBERAMERICA pretende convertirse, según el enunciado oficial, en un lugar de intercambio de los sectores más dinámicos de la sociedad civil de los 21 países comprometidos, y dará cabida a los proyectos que surjan desde la iniciativa CIBERMEDIA (que actuará como una línea de acción más dentro del propio programa Ibermedia).

\section{CONCLUSIONES}

En este contexto cultural y económico de circuitos globales y productos transnacionales, la comunidad iberoamericana tiene que incentivar medidas que puedan hacerla competitiva y diferenciada frente a otras regiones, ya que las últimas décadas, gracias a la oleada de liberalización y desprotección llevada a cabo por los estados nacionales, se ha convertido en terreno abonado y mercado inmejorable para las grandes empresas transnacionales del multimedia. No es de extrañar, por tanto, que pese a su tamaño y diversidad cultural ocupe un papel mínimo en el sector de las industrias culturales ${ }^{13}$ y que, especialmente, su industria audiovisual se haya visto monopolizada por los productos y empresas norteamericanos.

Como bien señala Néstor García Canclini (2004:195) “culturas internacionalmente más numerosas son arrinconadas hoy en lugares

13 Según los datos de la UNESCO Latinoamérica y el Caribe participan únicamente de $2,5 \%$ del comercio cultural mundial. 
minoritarios de los mercados globales". La lengua dentro de los contenidos audiovisuales, el origen de las películas, los contenidos de los canales de pago, o las empresas publicitarias y de distribución nos hablan de la debilidad que la producción autóctona tiene dentro de la región iberoamericana y que sólo podremos afrontar desde políticas que

Garanticen la diversidad y la interculturalidad en los circuitos transnacionales. Estamos en una época en que crece la aceptación de la multiculturalidad en la educación y en los derechos políticos, pero se estrecha la diversidad en las industrias culturales (García Canclini, 2002).

Pese a todo, si algo nos debe mantener la ilusión es la muestra de persistencia, calidad y creatividad que demuestran los profesionales iberoamericanos del sector. En este sentido, tanto el cine español como el latinoamericano cada vez están más reconocidos internacionalmente y sus producciones son valoradas como obras de gran ingenio que suplen la falta de recursos con la imaginación y la voluntad de sus profesionales. Prueba ilustrativa de esto la tenemos en los últimos éxitos del cine argentino, que pese a la quiebra sistemática de su industria provocada por la enorme crisis que sufrió a principios de siglo, ha conseguido que películas como El hijo de la novia, Nueve Reinas o La ciénaga hayan sido exportadas a todo el mundo. Encontramos igualmente algunos ejemplos de cine mexicano o brasileño que han conseguido este reconocimiento internacional, Amores perros, Central do Brasil o Cidade de Deus, serían algunos de los títulos más ilustrativos. ${ }^{14}$

Si tenemos este potencial creador y un enorme mercado interno, propuestas como la de Ibermedia resultan absolutamente necesarias para abrir nuevas vías de cooperación, nuevos espacios de producción,

14 Un caso especial en los últimos años (al menos de repercusión en España) ha sido el cine chileno, que a través de películas como El chacotero sentimental o Celebración ha conseguido por primera vez hacerse notar fuera de sus fronteras. Celebración, dirigida por Silvio Caiozzi, ganó en el 2000 el Colón de Oro en el Festival Iberoamericano de Huelva y, curiosamente, otro largometraje chileno, Taxi para tres de Osvaldo Lubertt, se imponía un año más tarde en la 49ª edición del Festival de San Sebastián. 
distribución y exhibición donde las producciones locales y los productores y realizadores independientes tengan la oportunidad de mostrar sus cualidades y de contar nuestras historias que, como lo demuestran las mejores producciones, pueden ser absolutamente populares, universales e imaginativas.

Pasados ya más de diez años desde su aprobación y con ocho de funcionamiento, Ibermedia ha contribuido, sin duda, a fortalecer los vínculos culturales de Iberoamérica, dinamizando un sector industrial clave y multiplicando su potencial a través del papel difusor que el discurso audiovisual juega en nuestras sociedades. De este modo, además del número de proyectos que han salido adelante, de la cantidad de empleos que se generaron, del impulso que ha supuesto para pequeños países y de la reactivación de las instituciones y de los profesionales del audiovisual, podemos decir que el programa está ayudando igualmente a construir una comunidad imaginada, pero cada día más visible y diferenciada dentro del contexto mundial.

Pese a todo este esfuerzo, aún queda un largo camino por recorrer hasta consolidar un verdadero espacio común, donde los países intercambien sus producciones, faciliten el acceso de los demás países integrantes o planteen medidas de protección para las industrias nacionales. Por consiguiente, es prioritario plantear normativas globales en las que participen todos los gobiernos de la región y en las que se recojan facilidades para la distribución, cuotas de pantalla en las salas de cine (como existe en la Comunidad Europea, aunque no siempre se cumplan), compromisos públicos y privados de apoyo a las coproducciones y políticas de subvención a los proyectos nacionales.

Esperemos, en este sentido, que Ibermedia haya sido tan sólo un primer paso, la piedra fundamental en, lo que Alberto García Ferrer (2006) denomina como la "apuesta razonada por hacer del audiovisual un horizonte en el que se pudieran sumar empeños comunes, potenciar los múltiples esfuerzos creativos y hacer visible la diversidad de Iberoamérica".

\section{Bibliografía}

ÁlVAREZ MONZONCILlO, José María: "Las transformaciones industriales en el cine mundial" en VVAA (1995) Historia General del Cine. volumen XII. Madrid: Cátedra. 
BUSTAMANTE, Enrique (1999) "Audiovisual y desarrollo regional: una perspectiva económica” en Ledo, M. y Krohling, M. (eds.) Comunicación audiovisual: investigación y formación universitarias. Santiago de Compostela: Universidad de Santiago de Compostela.

- (1996) «La comunicación en América Latina», Telos, núm 47, Madrid: Fundesco.

DraGo, Tito y Ruiz de Gopegui, Luis Ángel (eds.) (1991) Innovación tecnológica y comunicación: V Encuentro Iberoamericano de Comunicación, Mérida, 1990. Madrid: Extremadura Enclave 92 / Quinto Centenario.

FAPAE (1997) Diagnóstico de la industria audiovisual iberoamericana. Madrid: FAPAE.

FRAERMAN, Alicia (ed.) (1995) Identidad y nuevos medios: la comunicación en la utopía o realidad de Iberoamérica. Madrid: Comunica.

EUROPEAN AUDIOVISUAL OBSERVATORY. (2002) "Press note on the distribution of foreign films within the European Union (19962002)" disponible en http://www.obs.coe.int/about/oea/pr/disfilms_pays_tiers.html

GARCÍA CANCLINI, N. y Moneta, C. (eds.) (1999) Las industrias culturales en la integración latinoamericana. Buenos Aires: Eudeba.

GARCÍA CANCLINI, Néstor (2004) Diferentes, desiguales y desconectados. Mapas de la interculturalidad. Barcelona: Gedisa.

- (2002) "Economía y cultura: los países latinos en la esfera pública transnacional", disponible en www.campusoei.org/tres_espacios/ icoloquio11.htm

GARCÍA FERRER, Alberto (2006) “Iberoamérica, el avión y la cultura. ¿Por dónde empezar?”, Letra Internacional, núm 90, Madrid: Fundación Pablo Iglesias.

GETINO, Octavio (2002) "Economía y desarrollo en las industrias culturales de los países del Mercosur”. 23 Conferencia y Asamblea General de AIECS/IAMCR/AIERI Asociación Internacional de Estudios en Comunicación Social Barcelona, 21-26 de julio.

- (1998) "El mercado audiovisual latinoamericano", Voces y Culturas, núm. 13, Barcelona.

JONES, Daniel E."Investigaciones en España sobre la comunicación iberoamericana” en PCLA - volume 1 - número 3. São Paulo: Unversidad Metodista de São Paulo. 
LABRADA, Fernando (dir.) (1997) La industria audiovisual iberoamericana: datos de sus principales mercados. Madrid: AECI / FAPAE.

MARTÍN-BARBERO, Jesús (2002a) "Medios y culturas en el espacio latinoamericano", Iberoamericana. América Latina-España-Portugal. 6. Madrid: OEI.

- (2002b): "La globalización en clave cultural: Una mirada latinoamericana", ponencia presentada en el Coloquio Internacional Globalismo y Pluralismo celebrado en Montreal en abril de 2002.

ORTIZ, Renato (1994) Mundialização e cultura. São Paulo: Brasiliense.

PÉREZ HERRERO, P. y Tabanera, N. (coord.) (1993) España-América Latina: un siglo de politicas culturales. Madrid: Monografías Aieti.

RONCAGLIOLO, Rafael (1996) "La integración audiovisual en América

Latina: estados, empresas y productores independientes" en García Canclini, N. (coord.): Culturas en globalización. América Latina - Europa - Estados Unidos: libre comercio e integración. Caracas: Nueva Sociedad.

SÁNCHEZ RUIZ, Enrique E. (2001) “Globalización y convergencia: retos para las industrias culturales latinoamericanas" en Innovatec-Innovarium Inteligencia del Entorno C.A. Registro Mercantil VII núm 06087, tomo 83-A-VII número 62, Distrito Federal, Venezuela.

SIERRA CABAllERO, Francisco (2002) Bases de la política audiovisual europea. Sevilla: Mergablum.

TOLEDO, Teresa (ed.) (1998) Made in Spanish '98: la distribución y la exhibición en Iberoamérica. Editado en Donostia-San Sebastián por el Festival Internacional de Cine de Donostia-San Sebastián, S. A.

UNESCO (2000) "A survey on national cinematography", disponible en http://www.unesco.org/culture/industries/cinema/html_eng/survey. shtml.

SINCLAIR, John (1999) Latin American television: a global view. Oxford: Oxford University Press.

ZALLO, Ramón (1998) Economía de la comunicación y la cultura. Madrid: Akal. 